\title{
Corporate control and real investment in incomplete markets
}

\author{
Julien Hugonnier ${ }^{\mathrm{a}}$, Erwan Morellec ${ }^{\mathrm{b}, *}$ \\ ${ }^{a}$ Swiss Finance Institute, University of Lausanne, Switzerland \\ ${ }^{\mathrm{b}}$ Swiss Finance Institute and CEPR, Ecole des HEC, University of Lausanne, Rte de Chavannes 33, 1007 \\ Lausanne, Switzerland
}

Received 16 November 2004; accepted 7 September 2006

Available online 13 November 2006

\begin{abstract}
In the standard real options approach to investment under uncertainty, agents formulate optimal policies under the assumptions of risk neutrality or perfect capital markets. However, in most situations, corporate executives face incomplete markets either because they receive compensation packages that restrict their portfolios or because cash flows from the firm's investment opportunities are not spanned by those of existing assets. The present paper examines the impact of managerial risk aversion on investment decisions when the manager is exposed to idiosyncratic risk and faces the risk of a control challenge. In the paper, the investment policy selected by the manager reflects a trade-off between his incentives to reduce risk and the need to ensure sufficient efficiency to prevent control challenges. The analysis demonstrates that risk aversion induces the manager to speed up investment, leading to a significant erosion of the value of the option to wait.
\end{abstract}

(C) 2006 Elsevier B.V. All rights reserved.

JEL classification: G11; G12; G31

Keywords: Incomplete markets; Risk aversion; Real options

\footnotetext{
${ }^{*}$ Corresponding author. Tel./fax: +410216923357.

E-mail address: erwan.morellec@unil.ch (E. Morellec). 


\section{Introduction}

Since the seminal contributions of Brennan and Schwartz (1985) and McDonald and Siegel (1986), the literature analyzing investment decisions using the real option approach has developed substantially. ${ }^{1}$ One of the most cited result in this literature is that, with uncertainty and irreversibility, there exists a significant option value of waiting to invest. As a consequence, firms should only invest when the asset value exceeds the investment cost by a potentially large option premium. However, the assumptions of risk-neutrality or perfect capital markets on which this result relies are not particularly relevant to most real-world environments. While large shareholders may be able to perfectly diversify their wealth, corporate executives are typically exposed to idiosyncratic risk. ${ }^{2}$ As a result, their policy choices should reflect their attitude towards risk.

In this paper, we show how managerial risk aversion can affect investment policy and firm value when managers face incomplete markets. To make the intuition as clear as possible, we use a simple generalization of the standard McDonald and Siegel (1986) framework in which the manager is exposed to idiosyncractic risk and cannot undo this exposure by trading in the firm's assets. In that model, the firm has perpetual rights to a project and seeks to determine the investment date that maximizes the value of the project. In addition, "risk aversion by investors is introduced [...] by supposing that options to invest are owned by well-diversified investors, who need only be compensated for the systematic component of the risk of projects and options to invest.' Within the present paper, the decision maker faces incomplete markets and cannot eliminate idiosyncratic risk. Therefore, he requires compensation for both idiosyncratic and systematic risks.

The derivation of the results in the paper proceeds in two steps. The first step solves the portfolio policy of the manager and derives his indirect utility of wealth. The second step examines the impact of risk aversion and market incompleteness on investment decisions. To get conservative estimates of the distortions implied by managerial risk aversion, we allow the manager to reduce his risk exposure in two ways. First, the manager can invest in the market portfolio and a risk-free asset. Second, he can select the timing of investment, thereby affecting firm risk. In addition, we presume that when the policy choices of the manager erode firm value, the manager can face the threat of a control challenge and incur a cost that increases with deviations from value-maximization in investment policy.

The analysis in the paper reveals that when the manager has control rights over investment policy, his investment decisions reflect a trade-off between his incentives to invest early to reduce idiosyncratic risk and the need to ensure sufficient efficiency to prevent costly control transactions. The paper also demonstrates that managerial

\footnotetext{
${ }^{1}$ Dixit and Pindyck (1994) survey early real options models. Recent developments include the impact of competition (see Grenadier, 2002), financial leverage (Hennessy, 2004), learning (Descamps et al., 2004), liquidity (Mello and Parsons, 2000), macroeconomic conditions (Guo et al., 2005), and agency (Grenadier and Wang, 2005).

${ }^{2}$ Exposure to idiosyncratic risk may arise because the cash flows from the firm's projects are not spanned by those of existing assets, because of transaction costs, or because of compensation packages.
} 
risk aversion typically has a large impact on investment policy and project value. Specifically, the difference in net present values under firm- and utility-maximizing policies can reach $50 \%$ for reasonable parameter values. As shown in the paper, this reduction in NPVs arises because the manager has a strong incentive to invest early in the project, as manifested by his decision to select an investment threshold (minimum NPV) that is too low relative to the value-maximizing threshold. In particular, exposure to idiosyncratic risk quickly erodes the value of the option to wait and leads to investment near the zero NPV threshold.

The analysis in the present paper relates to two distinct strands of literature. First, from a modeling perspective, our analysis relates to the papers of Henderson (2005) and Miao and Wang (2005), in which the authors examine the impact of market incompleteness on investment decisions under uncertainty. In these papers, there are no agency conflicts between the decision maker and the owner of the project. In addition, the decision maker is characterized by an exponential utility function that precludes wealth effects. Importantly, while the analysis in Henderson is similar to ours, Miao and Wang introduce interesting differences by allowing the project owner to optimize his consumption stream and to hedge (partly) idiosyncratic shocks. Second, from an economic perspective, the paper relates to the growing literature that analyzes the implications of imperfect corporate governance for firms' policy choices (see e.g. Stulz, 1990, or Zwiebel, 1996). So far, theoretical work in this area has largely been qualitative, focusing only on directional effects (see however Morellec, 2004). Moreover, this literature has been mostly concerned with the size of investment, i.e. how much to invest, rather than with its timing, i.e. when to invest. The present paper encompasses elements of both literatures in a unified framework.

The remainder of the paper is organized as follows. Section 2 describes the model. Section 3 derives the relation between risk aversion and investment timing. Section 4 discusses implications. Section 5 concludes.

\section{Model and assumptions}

This paper analyzes the impact of managerial risk aversion on the firm's investment decisions when the manager has decision rights over investment policy and faces incomplete markets. For doing so, we consider an infinite horizon model of a continuous time economy where uncertainty is represented by a filtered probability space $(\Omega, \mathbb{F}, \mathscr{F}, \mathbb{P})$ on which are defined two independent standard Brownian motions $Z$ and $Z^{M}$. The filtration $\mathbb{F}:=\left(\mathscr{F}_{t}\right)_{t \geqslant 0}$ representing the information flow in this economy is the usual augmentation of the filtration generated by the Brownian motions.

\subsection{Investment opportunity set}

There are three assets available for continuous trading by unconstrained investors. The first security is a risk free bond with price process $B$ satisfying

$$
\mathrm{d} B_{t}=r B_{t} \mathrm{~d} t, \quad B_{0}=1,
$$


where $r>0$ is the risk-free rate. The other two assets are risky securities. The first risky asset is a market index. We denote its price process by $M$ and assume that

$$
\mathrm{d} M_{t}=\mu_{M} M_{t} \mathrm{~d} t+\sigma_{M} M_{t} \mathrm{~d} Z_{t}^{M}, \quad M_{0}>0,
$$

where $\mu_{M}$ is the constant expected rate of return on the market portfolio, $\sigma_{M}>0$ is the constant volatility of market returns and $Z^{M}$ represents market risk.

The second risky asset represents the stock of a firm in the market portfolio. We denote its price process by $P$ and assume that its dynamics are given by

$$
\mathrm{d} P_{t}=(r+\xi \theta) P_{t} \mathrm{~d} t+\xi P_{t} \mathrm{~d} Z_{t}^{M}+\zeta P_{t} \mathrm{~d} Z_{t}, \quad P_{0}>0,
$$

for some constants $\xi$ and $\zeta \neq 0$ where $\theta=\sigma_{M}^{-1}\left[\mu_{M}-r\right]$ is the market price of risk. The role of this second risky asset is to ensure that the securities available to unconstrained investors span both the market risk factor $Z^{M}$ and the idiosyncratic risk factor $Z$.

\subsection{Preferences and trading strategies}

The economy is populated with two types of agents: shareholders and the firm's manager. Shareholders are unconstrained and have access to the complete financial market described above. As a result, the preferences of these agents are irrelevant since they can perfectly hedge any risk by a controlled portfolio of the traded securities. By contrast, the manager is only allowed to trade in the bond and the market portfolio and thus faces incomplete markets since he cannot hedge against the variations of the idiosyncratic risk factor. We assume throughout the paper that the preferences of the manager are represented by the functional

$$
(T, X) \longmapsto \mathrm{E}\left[\mathrm{e}^{-\rho T} U(X)\right]=\mathrm{E}\left[\frac{\mathrm{e}^{-\rho T} X^{1-R}}{1-R}\right],
$$

for wealth $X$ available at time $T$. In this specification, the constant $R \in(0,1)$ is the manager's relative risk aversion and $\rho>0$ represents his subjective discount rate. ${ }^{3}$

We assume that the manager is endowed with an initial capital $x>0$ and denote by $\pi$ the proportion of his wealth that the manager invests in the market portfolio. Under the usual self-financing and integrability conditions (see Duffie, 2003), the proportion $1-\pi$ of the manager's wealth is invested in the risk-free security and the dynamics of the manager's wealth are given by

$$
\frac{\mathrm{d} X_{t}}{X_{t}}=\left(1-\pi_{t}\right) r \mathrm{~d} t+\pi_{t} \frac{\mathrm{d} M_{t}}{M_{t}}, \quad X_{0}=x .
$$

Given the fact that our horizon is potentially infinite, we define the set of admissible trading strategies for the manager as

$$
\mathscr{A}:=\left\{\pi: X_{\tau} \geqslant 0 \text { for all } \tau \in \mathscr{T}\right\},
$$

\footnotetext{
${ }^{3}$ Similar results obtain when $R>1$. However, the restrictions imposed on the parameter values have to be modified and the proof of our main result is much more involved.
} 
where $\mathscr{T}$ denotes the set of $\mathbb{F}$-stopping times. Using standard calculations, it is immediate to show that the discounted wealth process of an admissible strategy is a supermartingale under any risk-neutral probability measure, so that the set $\mathscr{A}$ is arbitrage-free.

\subsection{Investment policy and firm value}

We consider a setting in which shareholders delegate investment decisions to the manager, taking advantage of his skills (see also Grenadier and Wang, 2005). To keep the analysis as simple as possible, we assume that the firm's only asset is an option to invest the amount $I$ in a project paying a dividend $\delta A$ per unit of time for $\Delta$ years. In this specification $\delta$ is a strictly positive constant representing the rate at which dividends are paid and $A$ is the non-negative process defined by

$$
\mathrm{d} A_{t}=\mu_{A} A_{t} \mathrm{~d} t+\beta \sigma_{M} A_{t} \mathrm{~d} Z_{t}^{M}+\kappa A_{t} \mathrm{~d} Z_{t}, \quad A_{0}=a>0,
$$

for some constants $\beta$ and $\kappa>0$. In this equation, the constant $\kappa$ represents the residual variance of assets returns and $Z$ represents idiosyncratic risk. This Brownian motion is independent of the market risk factor $Z^{M}$ so that the covariance between the market index and the value of the firm's assets is fully captured by the parameter $\beta$.

We show below that it is optimal for the manager to consider investment policies under which the investment time $\tau$ is the first passage time of the underlying asset value process $A$ above a constant trigger level $\alpha$. In this case, the initial value of the firm to shareholders is given by

$$
S(\alpha)=(\alpha \phi-I)\left(\frac{a}{\alpha}\right)^{\Theta_{0}}
$$

for all $a \leqslant \alpha$ where we have set

$$
\phi:=\frac{\delta}{r+\beta \sigma_{M} \theta-\mu_{A}}\left[1-\mathrm{e}^{-\left(r+\beta \sigma_{M} \theta-\mu_{A}\right) \Delta}\right],
$$

and

$$
\Theta_{0}:=\frac{1}{2}-\frac{\mu_{A}-\beta \sigma_{M} \theta}{\kappa^{2}+\beta^{2} \sigma_{M}^{2}}+\sqrt{\left(\frac{1}{2}-\frac{\mu_{A}-\beta \sigma_{M} \theta}{\kappa^{2}+\beta^{2} \sigma_{M}^{2}}\right)^{2}+\frac{2 r}{\kappa^{2}+\beta^{2} \sigma_{M}^{2}}}>1 .
$$

If shareholders had control rights over the firm's investment decisions, they would choose the investment policy that maximizes the initial value $S(\alpha)$ of the firm. The corresponding investment policy consists in investing as soon as the value of the underlying assets exceeds the value maximizing threshold $\alpha_{0}$ defined by

$$
\alpha_{0}:=\frac{\Theta_{0}}{\Theta_{0}-1} \phi^{-1} I
$$

In our setting, shareholders delegate the investment decision to a manager. As a result, the firm's investment policy will reflect both the preferences of the manager 
towards risk and the impact of the market for corporate control on the policy choices of the manager. The next section describes this trade-off in greater detail.

\subsection{Agency costs and the market for corporate control}

We follow Zwiebel (1996) and Morellec (2004) by making the following two assumptions. First, we presume that the manager has control rights over investment policy. While in their settings this allocation of control rights implies that the manager chooses how much to invest, in our model it implies instead that the manager chooses when to invest. Second, we consider that the manager is constrained by the market for corporate control when making investment decisions. Notably, policies that imply deviations from value maximization expose the manager to the risk of a control transaction at the time of investment. We denote the probability of a costly control challenge by $\mathrm{p}(\alpha)$ where $\alpha$ denotes the value of the underlying assets at the time of investment. This probability increases with deviations from value maximization and is given by

$$
\mathrm{p}(\alpha)=1-1_{\{\alpha \phi \geqslant I\}}\left(\frac{S(\alpha)}{S\left(\alpha_{0}\right)}\right)^{\gamma},
$$

where $\gamma \in(0,1), \alpha$ is the investment threshold selected by the manager, $\alpha_{0}$ is the value maximizing investment threshold defined in (11), and $S($.$) is the value of the project$ defined in (8). In this specification, the parameter $\gamma$ represents the cost for the manager of deviating from value-maximization. Importantly, the probability of a control challenge increases with agency costs, defined as the reduction in the value of the project associated with managerial discretion. In particular, $\mathrm{p}(\alpha)=0$ and there is no risk of a control challenge if the manager implements the value maximizing investment policy.

The manager's wealth at the time of investment depends on the selected investment policy in the following way. If the manager is not replaced at the time of investment, his wealth is scaled up by a factor $b>1$. If, on the other hand, he is replaced, then his wealth is scaled down by the non-negative factor

$$
f(\alpha)= \begin{cases}c\left(\frac{\alpha \phi}{I}\right)^{\eta} & \text { if investment has a negative NPV, i.e. if } \alpha \phi \leqslant I, \\ c & \text { otherwise, }\end{cases}
$$

where the parameter $c \in(0,1)$ represents the fraction of his wealth that the manager retains upon being replaced and $\eta \geqslant 1$ is a cost parameter. This specification shows that if the manager is replaced, he incurs a negative shock to his wealth (for example due to reputation). The reduction in wealth is more important if the manager has invested in a negative NPV project and increases as the project's NPV decreases. This specification also implies that at the time $\tau$ of investment the manager's incurs a proportional shock to his wealth which is given by

$$
\vartheta_{\tau}:=f\left(A_{\tau}\right)+\mathbf{1}_{\mathscr{B}_{\tau}}\left[b-f\left(A_{\tau}\right)\right],
$$

where $\mathscr{B}_{\tau}$ denotes the set of states of nature in which the manager is not replaced.

We now turn to the analysis of investment decisions. 


\section{Risk aversion and investment timing}

The manager's objective is to maximize the discounted utility of his wealth by selecting his portfolio strategy $\pi$ as well as the firm's investment time $\tau$. We can thus write his indirect utility function as

$$
u(x, a):=\sup _{\tau \in \mathscr{T}} u(x, a \mid \tau)=\sup _{\tau \in \mathscr{T}} \sup _{\pi \in \mathscr{A}} \mathrm{E}\left[\mathrm{e}^{-\rho \tau} U\left(\vartheta_{\tau} X_{\tau}^{\pi}\right)\right],
$$

where $X^{\pi}$ is the wealth process associated with the admissible trading strategy $\pi \in \mathscr{A}$ and $\vartheta$ is the shock to manager's wealth at the time of investment $\tau$ (defined in (14)).

Let $\mathbb{F}^{a}:=\left(\mathscr{F}_{t}^{a}\right)$ denote the usual augmentation of the filtration generated by the underlying asset value process. The manager's dynamic budget constraint being linear and his utility being homogenous of degree $1-R$ in wealth, we have that the identity $u(x, a \mid \tau)=x^{1-R} u(1, a \mid \tau)$ holds true for all initial levels of capital $x>0$. As a result, the value of the underlying asset is the only relevant state variable for the optimal stopping problem and we may restrict ourselves to the set $\mathscr{T}^{a}$ of $\mathbb{F}^{a}$-stopping times when computing the first supremum in Eq. (15).

To facilitate the exposition of our results, we first fix some notation. Let $(\lambda, \Phi, q)$ be the constants defined by

$$
\begin{aligned}
& \lambda:=\left(\frac{1-R}{R}\right) \theta, \\
& \Phi:=1+\frac{1-R}{R}\left(\frac{\beta^{2} \sigma_{M}^{2}}{\kappa^{2}+\beta^{2} \sigma_{M}^{2}}\right)>1, \\
& q:=\Phi\left[\rho-(1-R)\left(r+\frac{\theta^{2}}{2 R}\right)\right],
\end{aligned}
$$

and denote by $\Theta_{1}$ the non-negative solution to the quadratic equation

$$
\left(\mu_{A}+\beta \sigma_{M} \lambda\right) \Theta+\frac{1}{2} \Theta(\Theta-1)\left(\kappa^{2}+\beta^{2} \sigma_{M}^{2}\right)-q=0 .
$$

Furthermore, let $\overline{\mathrm{P}}$ be the equivalent probability measure defined by

$$
\left.\frac{\mathrm{d} \overline{\mathrm{P}}}{\mathrm{dP}}\right|_{\mathscr{F}_{t}}:=\exp \left[\lambda Z_{t}^{M}-\frac{1}{2} \lambda^{2} t\right]
$$

and denote the associated expectation operator by $\overline{\mathrm{E}}$. The following theorem provides a complete solution to the manager's portfolio choice problem given a fixed investment policy (i.e. given an arbitrary stopping time $\tau \in \mathscr{T}^{a}$ ).

Theorem 1. Assume that the constant $q$ is strictly positive and let $\tau$ be an arbitrary stopping time in the underlying assets' filtration. Then, for any given investment policy $\tau$, the manager's discounted utility of wealth satisfies

$$
u(x, a \mid \tau)=U(x) \overline{\mathrm{E}}\left[\mathrm{e}^{-q \tau} \vartheta_{\tau}^{(1-R) \Phi}\right]^{1 / \Phi} .
$$


Having obtained an explicit solution for the value function of the manager's portfolio choice problem, we can now turn to the determination of the utilitymaximizing investment policy. Using the result of Theorem 1 in conjunction with the law of iterated expectations and the definition of $\mathscr{B}_{\tau}$, we have that the manager's indirect utility function can be written as

$$
u(x, a)=U(x)\left\{\sup _{\tau \in \mathscr{T}^{a}} \overline{\mathrm{E}}\left[\mathrm{e}^{-q \tau} h\left(A_{\tau}\right)\right]\right\}^{1 / \Phi}
$$

where the deterministic reward function $h$ is defined by

$$
h(\alpha):=\overline{\mathrm{E}}\left[\vartheta_{\tau}^{(1-R) \Phi} \mid A_{\tau}=\alpha\right]=b^{(1-R) \phi}+\mathrm{p}(\alpha)\left[f(\alpha)^{(1-R) \Phi}-b^{(1-R) \Phi}\right] .
$$

This maximization problem is similar to traditional maximization problems in the real options literature since the objective function is equal to the product of a reward function and a stochastic discount factor. The main difference between our setting and traditional models is that in our formulation markets are incomplete and the manager is subject to the possibility of a control challenge. This results in endogenous distortions of the reward function, the discount rate and the valuation measure and implies that the investment threshold selected by the manager depends on his degree of relative risk aversion.

The manager's reward function $h$ being strictly increasing on the interval $\left[0, \alpha_{0}\right]$ and strictly decreasing otherwise, it is natural to conjecture that the utilitymaximizing policy consists in investing as soon as the value of the underlying assets reaches a critical level $\alpha^{*} \in\left[\phi^{-1} I, \alpha_{0}\right]$. The following theorem makes this intuition precise and provides an explicit characterization of the investment policy selected by the manager.

Theorem 2. Assume that $q$ is strictly positive, that $\Theta_{1}<\eta(1-R) \Phi$, and that the starting value of assets is below the investment threshold selected by the manager. Then, the manager's indirect utility function is given by

$$
u(x, a)=U(x) \overline{\mathrm{E}}\left[\mathrm{e}^{-q \tau^{*}} h\left(A_{\tau^{*}}\right)\right]^{1 / \Phi}=U(x)\left[h\left(\alpha^{*}\right)\left(\frac{a}{\alpha^{*}}\right)^{\Theta_{1}}\right]^{1 / \Phi},
$$

where the constant $\alpha^{*}$ is the unique solution in the interval $\left[\phi^{-1} I, \alpha_{0}\right]$ to the non-linear equation:

$$
\Theta_{1}-\frac{a h^{\prime}(a)}{h(a)}=0
$$

and the optimal investment time $\tau^{*}$ is the first hitting time of the constant investment threshold $\alpha^{*}$ by the value of the underlying assets.

Having obtained a complete description of the manager's optimal investment strategy, we now turn to the analysis of the impact of risk aversion, incomplete markets and the market for corporate control on investment decisions. Since the 
Table 1

Input parameter values

\begin{tabular}{ll}
\hline Parameter & Value \\
\hline Risk free rate & $r=0.03$ \\
Dividend yield & $\delta=0.03$ \\
Market volatility & $\sigma_{M}=0.3$ \\
Idiosyncratic volatility & $\kappa=0.1$ \\
Sharpe ratio and beta & $\theta=0.3$ \\
Project beta & $\beta_{M}=1$ \\
Risk aversion coefficient & $R=0.5$ \\
Subjective discount factor & $\rho=0.15$ \\
Shocks to the wealth & $b=1.1$ and $c=0.5$ \\
Cost parameters & $\gamma=0.5$ and $\eta=3$ \\
Cost of investment & $I=1$ \\
\hline
\end{tabular}

This table reports the parameter values used in the base case environment. The subjective discount rate of the manager satisfies the transversality condition $\rho>(1-R)\left(r+\theta^{2} / 2 R\right)$. In addition, the cost parameter $\eta$ is such that $\Theta_{1}<\eta(1-R) \Phi$ and we set the drift of the underlying asset process equal to: $\mu_{A}=r-\delta+\beta \sigma_{M} \theta$.

investment threshold is the solution to a non-linear equation that does not admit an explicit solution, most of the results will be illustrated by numerical examples. ${ }^{4}$

\section{Model implications}

To determine the values of the quantities of interest, it is necessary to select parameter values for the initial value of the firm's assets $A_{0}$, the cost of investment $I$, the risk free interest rate $r$, the dividend yield $\delta$, the firm's beta $\beta_{M}$, the market volatility $\sigma_{M}$, idiosyncratic volatility $\kappa$, the subjective discount rate $\rho$, the Sharpe ratio $\theta$, the cost parameter $\gamma$, and the coefficient of risk aversion $R$. Whenever possible, we use the parameters values of Boyle and Guthrie (2003). Additional parameter values for the base case environment are reported in Table 1.

The model developed above yields a number of novel implications regarding investment decisions under uncertainty. These implications are grouped in two categories as follows.

\subsection{Risk aversion and the erosion of the option value to wait}

One of the major contributions of the real options literature is to show that with uncertainty and irreversibility, there exists a value of waiting to invest and the decision maker should only invest when the asset value exceeds the investment cost

\footnotetext{
${ }^{4}$ In Appendix C, we consider a reduced-form version of the model that generates closed-form solutions for the investment threshold selected by the manager. The numerical results associated with this alternative specification are quantitatively very close and qualitatively identical to those presented below.
} 
by a potentially large option premium. This effect is well summarized by Dixit and Pindyck (1994):

We find that [...] the option value [of waiting] is quantitatively very important. Waiting remains optimal even though the expected rate of return on immediate investment is substantially above the interest rate or the normal rate of return on capital. Return multiples as much as two or three times the normal rate are typically needed before the firm will exercise its option and make the investment.

Although this investment policy is consistent with what shareholders would want the firm to do, it is not consistent with what managers will do when taking into account risk aversion. Notably, Theorem 2 and the simulation results reported below show that the risk-averse manager has an incentive to speed up investment in comparison with the value-maximizing policy for well-diversified shareholders. To better understand this incentive to invest early, one has to recall that within the present model the wealth of the manager depends on the value of the asset underlying the project. As a result, the manager is exposed to idiosyncratic risk. By investing, the manager reduces his exposure idiosyncratic volatility, thereby increasing his discounted utility of wealth. This provides the manager with an additional benefit to investment and thus with an incentive to select an investment threshold (minimum NPV) that is too low relative to the value-maximizing threshold. This incentive is only partially offset by the impact of the market for corporate control on the policy choices of the manager, leading to early investment.

We can investigate the impact of risk aversion on the timing of investment by analyzing the change in the selected investment threshold due to managerial risk aversion. Fig. 1 plots the investment threshold selected by the firm if it were to follow the zero-NPV rule (short dashed line), the investment rule that maximizes shareholders' wealth (long dashed line) or the decision rule that maximizes managerial utility (solid line). In this figure, the investment threshold is represented as a function of (i) market volatility $\sigma_{M}$, (ii) idiosyncratic volatility $\kappa$, (iii) the risk aversion coefficient $R$, (iv) the subjective discount rate $\rho$, (v) the positive shock $b$, and (vi) the cost of a control challenge $\gamma$. For each simulation, we vary the value of one parameter. The other parameter values are set as in the base case environment.

Consistent with economic intuition, the figure shows that risk aversion tends to decrease the investment threshold selected by the manager, and hence to speed up investment. One direct implication of this result is that risk aversion significantly erodes the value of waiting to invest. Simulation results also reveal that when riskaverse managers have control rights over investment policy, the traditional NPV rule becomes increasingly descriptive as risk aversion or the manager's subjective discount rate increase. In other words, conflicts of interests between shareholders and the manager tend to mitigate some of the conclusions reached under perfect contracting.

Fig. 1 also reveals that when risk aversion is at play, market volatility and idiosyncratic volatility can have very different effects on the selected investment policy. In particular, the figure reveals that the larger market (i.e. priced) volatility, the larger the option value of waiting and the larger the selected investment 

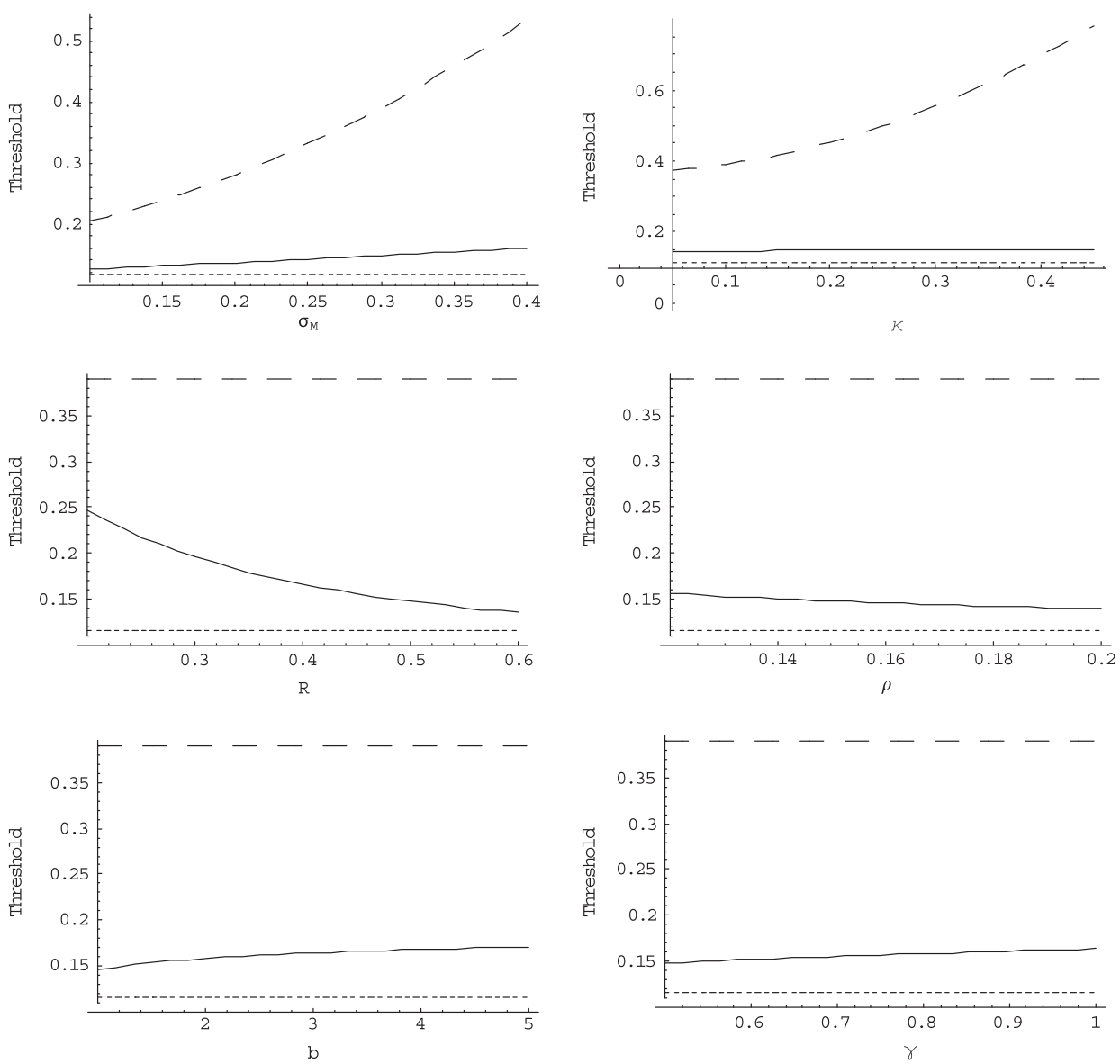

Fig. 1. Investment thresholds. The figure plots the investment threshold selected by the firm if it were to follow the zero-NPV rule (short dashed line), the investment rule that maximizes shareholders' wealth (long dashed line) or the decision rule that maximizes managerial utility (solid line). In this figure, the investment threshold is represented as a function of (i) market volatility $\sigma_{M}$, (ii) idiosyncratic volatility $\kappa$, (iii) the risk aversion coefficient $R$, (iv) the subjective discount rate $\rho$, (v) the positive shock $b$, and (vi) the cost of a control challenge $\gamma$. For each simulation, we vary the value of one parameter. The other parameter values are set as in the base case environment.

threshold. By contrast, an increase of idiosyncratic volatility imposes more (unpriced) risk on the manager so that idiosyncratic volatility has little or no impact on the selected investment policy. The figure also shows that the market for corporate control can have a large impact on the firm's investment policy. Notably, the investment threshold selected by the manager gets closer to the value-maximizing one as the cost of a control challenge $\gamma$ increases.

The impact of managerial risk-aversion on investment policy can also be analyzed by examining the change in the probability of investment due to risk aversion. 
The probability of investment over the time interval $[0, T]$ is given by

$$
\mathrm{P}=\mathscr{N}\left[\frac{-\ln (y / a)+m T}{\sqrt{\left(\kappa^{2}+\beta^{2} \sigma_{M}^{2}\right) T}}\right]+\left(\frac{y}{a}\right)^{2 m /\left(\kappa^{2}+\beta^{2} \sigma_{M}^{2}\right)} \mathscr{N}\left[\frac{-\ln (y / a)-m T}{\sqrt{\left(\kappa^{2}+\beta^{2} \sigma_{M}^{2}\right) T}}\right],
$$

where $m=\mu_{A}-\left(\kappa^{2}+\beta^{2} \sigma_{M}^{2}\right) / 2, \mathscr{N}$ is the standard normal cumulative distribution function, and $y$ is the investment threshold.

In the base case environment, the probability of investment over a 10 year horizon is $28 \%$ under the value-maximizing investment policy and $79 \%$ under the utilitymaximizing investment policy. Thus, managerial risk aversion favors investment and has a significant impact on the likelihood of investment. To get more insights on the impact of the various parameters of the model, Fig. 2 plots the probability of investment under the value-maximizing (dashed line) and utility-maximizing (solid line) investment policies as a function of (i) market volatility $\sigma_{M}$, (ii) idiosyncratic volatility $\kappa$, (iii) the risk aversion coefficient $R$, (iv) the subjective discount rate $\rho$, (v) the positive shock $b$, and (vi) the cost of a control challenge $\gamma$. For each simulation, we vary the value of one parameter. The other parameter values are set as in the base case environment. In this figure, a value of 0.4 for means that there is a $40 \%$ probability of investment over the 10-year horizon.

Consider first the impact of volatility. An increase in volatility (either through $\sigma_{M}$, $\kappa$, or $\beta$ ) increases the variability of the return on investment and therefore the value of waiting to invest for the manager. At the same time, an increase in volatility increases the potential fluctuations of the value process $A_{t}$, making it more likely that any given threshold will be reached over a given horizon. Fig. 3 reveals that for welldiversified shareholders (dashed line) the first effect always typically dominates so that the probability of investment decreases with volatility. As shown in Fig. 1, the impact of volatility on the selected threshold for a risk-averse manager is not as important as it is for a well-diversified shareholder. It can even be negative as far as idiosyncratic volatility is concerned. The natural consequence of this effect is that an increase in idiosyncratic volatility increases the likelihood of investment when investment decisions are made by risk-averse managers. Finally, Fig. 3 shows that as the benefit of investing early increases (either because $R$ increases or because $\rho$ increases), the likelihood of investment over a finite horizon increases. By contrast, as the cost of investing early increases (because $\gamma$ increases), the likelihood of investment over a finite horizon decreases.

\subsection{Agency costs and utility-maximization}

The above analysis shows that a risk-averse manager has an incentive to speed up investment in comparison with the value-maximizing policy for well-diversified shareholders. As a result, managerial discretion induces agency costs that are equal to the difference between current firm (option) values under firm- and utilitymaximizing policies. As a proportion of the value under the utility-maximizing 

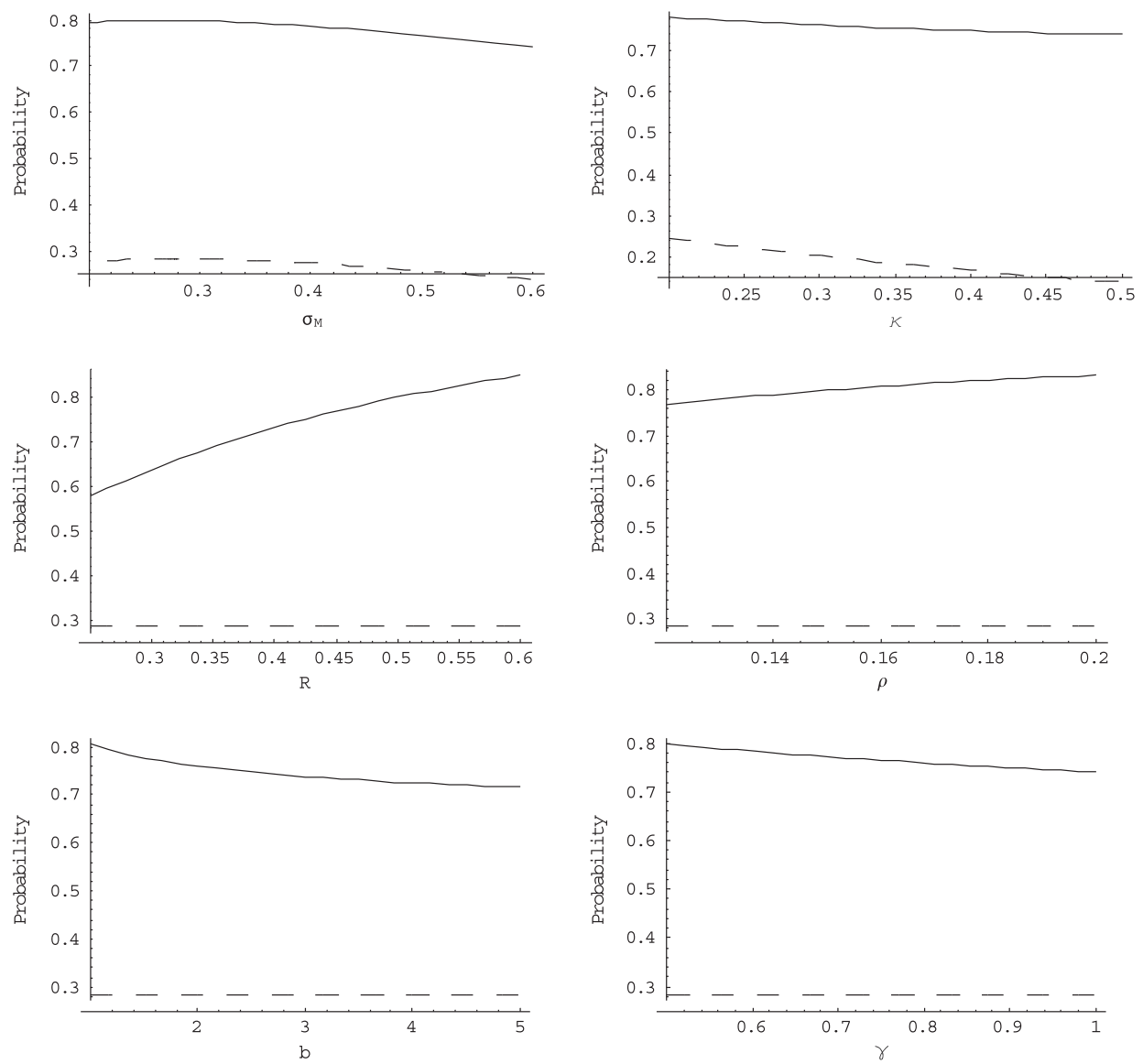

Fig. 2. Probability of investment. The figure plots the probability of investment under the valuemaximizing (dashed line) and utility-maximizing (solid line) investment policies as a function of (i) market volatility $\sigma_{M}$, (ii) idiosyncratic volatility $\kappa$, (iii) the risk aversion coefficient $R$, (iv) the subjective discount rate $\rho$, (v) the positive shock $b$, and (vi) the cost of a control challenge $\gamma$. For each simulation, we vary the value of one parameter. The other parameter values are set as in the base case environment. In this figure, a value of 0.4 for means that there is a $40 \%$ probability of investment over the 10 -year horizon.

policy, these agency costs are equal to

$$
\frac{S\left(\alpha^{*}\right)}{S\left(\alpha_{0}\right)}-1=\frac{\phi \alpha^{*}-I}{\phi \alpha_{0}-I}\left(\frac{\alpha_{0}}{\alpha^{*}}\right)^{\Theta_{0}}-1,
$$

where $\alpha^{*}$ is the investment threshold selected by the manager and $\alpha_{0}$ is the valuemaximizing investment threshold.

In the base case environment, agency costs amount to 55\% of project's NPV. Thus, managerial risk aversion encourages investment and has a significant impact on the NPV of investment projects. To get more insights on the impact of the various 

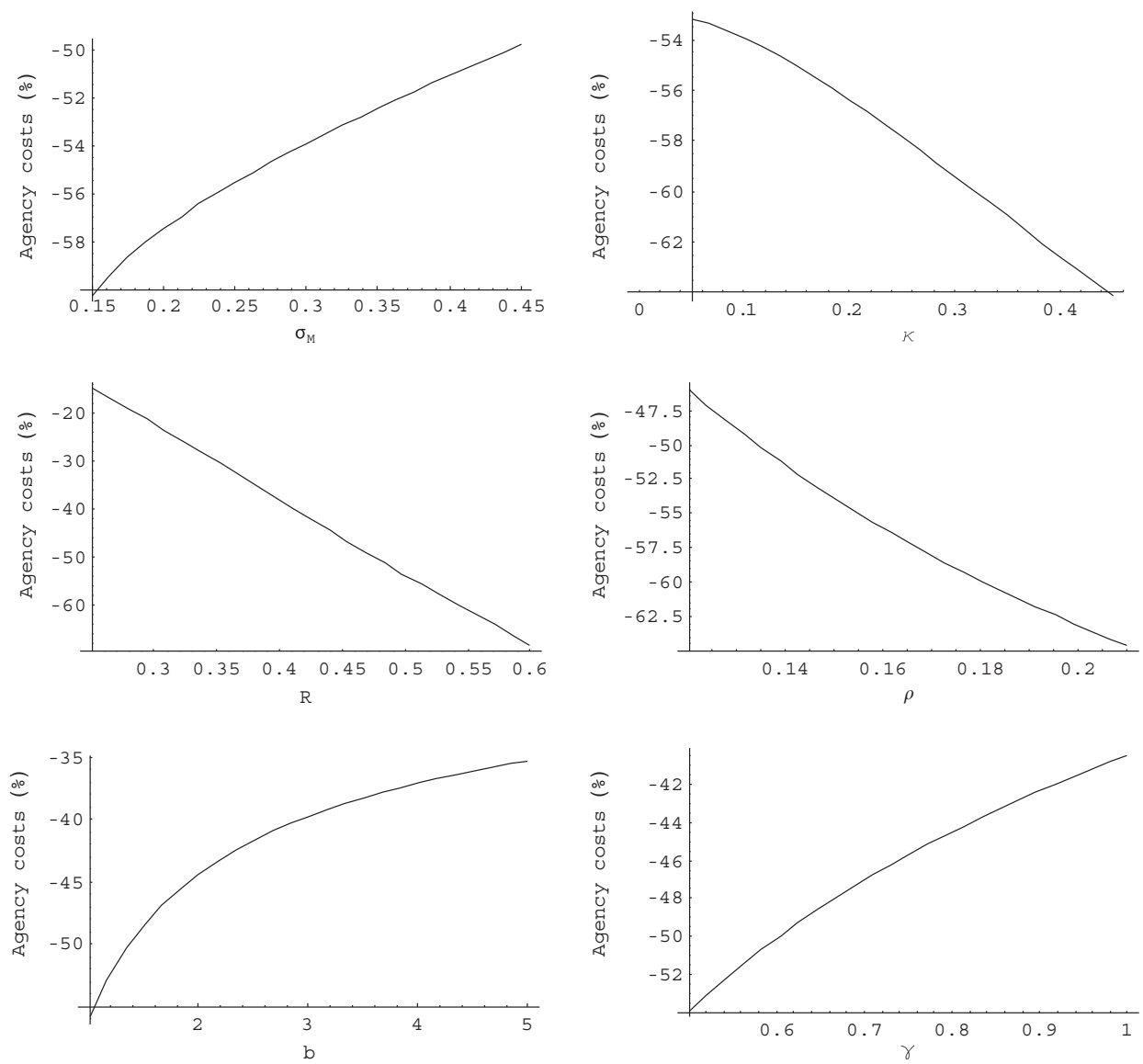

Fig. 3. Agency costs. The figure plots agency costs as a function of (i) market volatility $\sigma_{M}$, (ii) idiosyncratic volatility $\kappa$, (iii) the risk aversion coefficient $R$, (iv) the subjective discount rate $\rho$, (v) the positive shock $b$, and (vi) the cost of a control challenge $\gamma$. For each simulation, we vary the value of one parameter. The other parameter values are set as in the base case environment. In this figure, a value of -40 for means that managerial discretion reduces the NPV of the option to invest by $40 \%$.

parameters of the model, Fig. 3 plots agency costs as a function of (i) market volatility $\sigma_{M}$, (ii) idiosyncratic volatility $\kappa$, (iii) the risk aversion coefficient $R$, (iv) the subjective discount rate $\rho,(\mathrm{v})$ the positive shock $b$, and (vi) the cost of a control challenge $\gamma$.

Fig. 3 confirms the above intuition regarding agency costs. In particular, the model predicts that agency costs should increase with idiosyncratic volatility, the coefficient of risk aversion, and the subjective discount rate of the investor, and decrease with the cost $\gamma$ of a control challenge. Interestingly, agency costs decrease as systematic (priced) risk increases. These results illustrate that in addition to systematic risk, idiosyncratic risk is an important factor in the decision making 
process. Finally, the figure reveals that agency costs can raise sharply if one increases idiosyncratic risk or the coefficient of risk aversion.

Finally, and as a matter of completeness, Fig. 4 plots the expected utility of the manager under the utility-maximizing investment policy (solid line) and the project value-maximizing investment policy (dashed line).

This figure shows that there can be significant costs to the manager of following the value-maximizing investment policy. Again these costs increase with the risk aversion coefficient or idiosyncratic volatility and decrease with the probability of a control challenge and the cost of a control challenge.
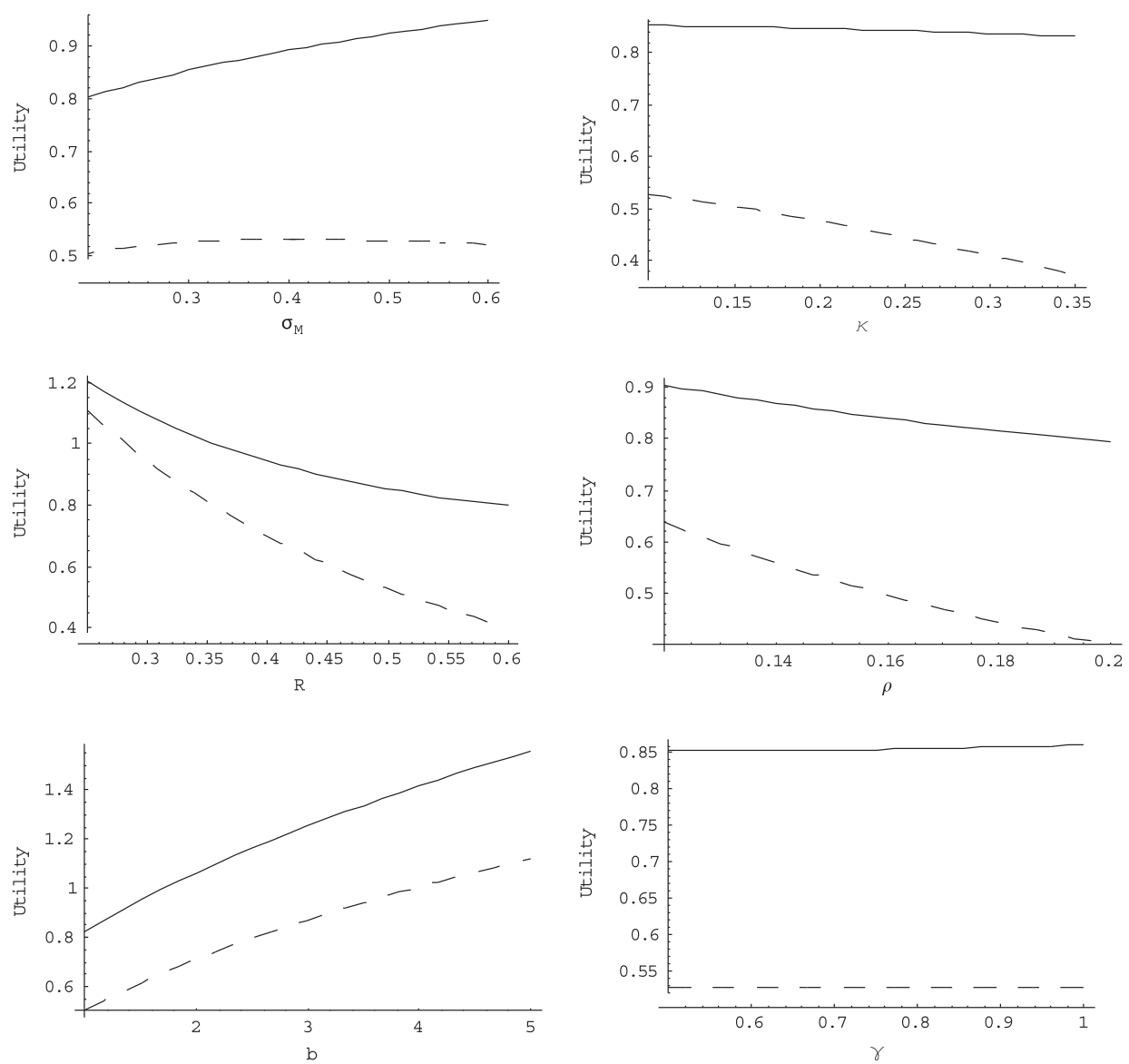

Fig. 4. Indirect utility and the timing of investment. The figure plots the indirect utility of the manager as a function of (i) market volatility $\sigma_{M}$, (ii) idiosyncratic volatility $\kappa$, (iii) the risk aversion coefficient $R$, (iv) the subjective discount rate $\rho,(\mathrm{v})$ the positive shock $b$, and (vi) the cost of a control challenge $\gamma$. For each simulation, we vary the value of one parameter. The other parameter values are set as in the base case environment. The solid line represents indirect utility under the utility maximizing investment policy $\alpha^{*}$. The dashed line represents indirect utility under the project value maximizing investment policy $\alpha_{0}$. 


\section{Conclusion}

Since the seminal papers by Brennan and Schwartz (1985), and McDonald and Siegel (1986), investment decisions under uncertainty have been the subject of considerable research in financial economics. In the vast majority of these models, it is either assumed that agents are risk neutral or that markets are complete, so that decisions are made in a preference-free environment. Yet, in most situations, managers face incomplete markets either because the cash flows from the firm's projects are not spanned by those of existing assets or because of compensations packages that restrict their portfolios. In such circumstances, we can expect risk aversion to play a role in investment decisions.

The present paper demonstrates that risk aversion provides an incentive for managers to speed up investment. As shown in the paper, this incentive to invest early erodes the value of waiting to invest, leading to investment near the zero NPV threshold. It also significantly increases the probability of investment over a given horizon. For example, in the base case, the probability of investment over a 10 -year horizon is $28 \%$ under the value-maximizing investment policy and 79\% under the utility-maximizing investment policy. The analysis also shows that these distortions in investment policy induce large agency costs, which can completely eliminate a project's NPV.

While the present paper focuses on the impact of market incompleteness on investment policy, similar results should obtain in other environment in which corporate executives cannot eliminate their exposure to idiosyncratic risk. For example, to the extent that the value of human capital is firm specific, managerial risk aversion may have an impact on firm policy choices even if the firm does not have an explicit compensation policy. Also, executives that have positions in non-traded companies will typically find it difficult to eliminate their exposure to firm risk. In such environments, one should expect risk aversion to speed up investment, the option value of waiting to be small, and firms to invest close to the zero NPV threshold.

\section{Acknowledgments}

This article builds on a previous paper titled "Investment under Uncertainty and Incomplete Markets". We thank Mike Barclay, Suleyman Basak, Pierre CollinDufresne, Denis Gromb, Ludger Hentschel, John Long, Michelle Lowry, Philip Schönbucher, Cliff Smith, Neng Wang, Jerry Zimmerman, the referee, and seminar participants at LBS and the University of Rochester for helpful comments and NCCR FINRISK of the Swiss National Science Foundation for financial support.

\section{Appendix A. Proof of Theorem 1}

Let $\tau$ denote an arbitrary stopping time of the underlying assets filtration and consider the pair of non-negative, bounded processes defined by

$$
H_{t}:=\overline{\mathrm{E}}\left[\mathrm{e}^{-q(\tau-t)} \vartheta_{\tau}^{(1-R) \Phi} \mid \mathscr{F}_{t}^{a}\right]^{1 / \Phi}=\left(\mathrm{e}^{q t} Q_{t}\right)^{1 / \Phi} .
$$


As is easily seen, the process $Q$ is a $\left(\mathbb{F}^{a}, \overline{\mathrm{P}}\right)$-martingale. On the other hand, it follows from well-known filtering results that the underlying assets' filtration coincides with the filtration of the one-dimensional standard $\overline{\mathrm{P}}$-Brownian motion

$$
\bar{W}_{t}:=\frac{\kappa Z_{t}}{\sqrt{\kappa^{2}+\beta^{2} \sigma_{M}^{2}}}+\frac{\beta \sigma_{M}\left[Z_{t}^{M}-\lambda t\right]}{\sqrt{\kappa^{2}+\beta^{2} \sigma_{M}^{2}}} .
$$

Thus, it follows from the martingale representation theorem that there exists an almost surely square integrable process $\phi$ such that

$$
Q_{t}=\overline{\mathrm{E}}\left[\mathrm{e}^{-q \tau} \vartheta_{\tau}^{(1-R) \Phi}\right]+\int_{0}^{t} \phi_{s} \mathrm{~d} \bar{W}_{s}
$$

Applying Itô's lemma to the right-hand side of Eq. (A.1) and using the definition of the process $\bar{W}$ we obtain that

$$
\mathrm{d} H_{t}=\left[\frac{q H_{t}}{\Phi}-\frac{1-R}{R}\left\{\psi_{t} \theta+\frac{\left|\psi_{t}\right|^{2}}{2 H_{t}}\right\}\right] \mathrm{d} t+\psi_{t} \mathrm{~d} Z_{t}^{M}+\xi_{t} \mathrm{~d} Z_{t},
$$

where the almost surely square integrable processes $(\psi, \xi)$ are defined by

$$
\left(\psi_{t}, \xi_{t}\right):=\left(\frac{\beta \sigma_{M}}{\sqrt{\kappa^{2}+\beta^{2} \sigma_{M}^{2}}} \frac{\phi_{t} H_{t}}{Q_{t}}, \frac{\kappa}{\sqrt{\kappa^{2}+\beta^{2} \sigma_{M}^{2}}} \frac{\phi_{t} H_{t}}{Q_{t}}\right) .
$$

Now let $\pi \in \mathscr{A}$ be an arbitrary admissible strategy for the manager and denote the corresponding wealth process by $X^{\pi}$. Applying Itô's lemma to the non-negative process

$$
Y_{t}^{\pi}:=\mathrm{e}^{-\rho t} U\left(X_{t}^{\pi}\right) H_{t}
$$

and using the homogeneity of the manager's utility function in conjunction with Eqs. (5) and (A.2), we obtain that

$$
\mathrm{d} Y_{t}^{\pi}=-Y_{t}^{\pi} \frac{1-R}{R}\left[\theta-R \pi_{t} \sigma_{M}+\frac{\psi_{t}}{H_{t}}\right]^{2} \mathrm{~d} t+\psi_{t}^{\pi} \mathrm{d} Z_{t}^{M}+\xi_{t}^{\pi} \mathrm{d} Z_{t},
$$

holds for some almost surely square integrable processes $\left(\psi^{\pi}, \xi^{\pi}\right)$. This implies that the process $Y^{\pi}$ is a local supermartingale, and hence also a global supermartingale since it is non-negative. The optional sampling theorem then implies that we have the inequality $\mathrm{E}\left[Y_{\tau}^{\pi}\right] \leqslant Y_{0}^{\pi}=U(x) H_{0}$, and it now follows from Eq. (A.1) and the arbitrariness of the trading strategy that

$$
u(x, a \mid \tau)=\sup _{\pi \in \mathscr{A}} \mathrm{E}\left[Y_{\tau}^{\pi}\right] \leqslant Y_{0}^{\pi}=U(x) H_{0} .
$$

Now consider the admissible trading strategy defined by

$$
\pi_{t}^{*}:=\frac{\theta}{\sigma_{M} R}+\frac{\psi}{\sigma_{M} H_{t} R} .
$$

As is easily seen from Eq. (A.3), we have that the process $Y^{*}:=Y^{\pi^{*}}$ is a local martingale since its drift is equal to zero. Admitting for the moment that this process 
is a martingale when restricted to the stochastic interval $[0, \tau]$, we obtain that

$$
u(x, a \mid \tau) \geqslant \mathrm{E}\left[Y_{\tau}^{*}\right]=Y_{0}^{*}=U(x) H_{0} .
$$

This contradicts Eq. (A.4) and hence establishes the optimality of $\pi^{*}$ as well as the expression for the manager's discounted utility of wealth given in the statement.

To complete the proof, we need to show that the process $Y^{*}$ is a uniformly integrable martingale on the interval $[0, \tau]$. Since both $q$ and $\Phi$ are strictly positive by assumption, there exists a strictly positive constant $\varepsilon<R(1-R)^{-1}$ such that

$$
p:=\frac{q}{\Phi}-\frac{\varepsilon(1-R)^{2}}{R-R(1+\varepsilon)(1-R)}>0 .
$$

On the other hand, using the boundedness of the process $\vartheta$ in conjunction with Eq. (5), we obtain that there exists a strictly positive constant $C$ and a non-negative local martingale $M^{\varepsilon}$ such that

$$
\left(Y_{\tau \wedge \zeta}^{*}\right)^{1+\varepsilon} \leqslant C\left(\mathrm{e}^{-\rho(\tau \wedge \zeta)} U\left(X_{\tau \wedge \zeta}^{*}\right)\right)^{1+\varepsilon} \leqslant C\left(\mathrm{e}^{-\mathrm{p}(\tau \wedge \zeta)} M_{\tau \wedge \zeta}^{\varepsilon}\right)
$$

holds for all stopping times $\zeta \in \mathscr{T}$. The constant $\mathrm{p}$ being strictly positive by construction, this implies that the non-negative local martingale $Y^{*}$ is uniformly integrable on the stochastic interval $[0, \tau]$ and our proof is complete.

\section{Appendix B. Proof of Theorem 2}

Straightforward computations using the definition of the function $h$ and the assumptions of the statement show that we have

$$
\sup _{\alpha \in \mathbb{R}_{+}}\left\{\alpha^{-\Theta_{1}} h(\alpha)\right\}=\left\{\left(\alpha^{*}\right)^{-\Theta_{1}} h\left(\alpha^{*}\right)\right\},
$$

where the constant $\alpha^{*}$ is defined as in the statement of the theorem. On the other hand, applying Itô's lemma and using the definition of the constants $q$ and $\Theta_{1}$ shows that

$$
L_{t}:=\mathrm{e}^{-q t} A_{t}^{\Theta_{1}}
$$

is a non-negative local martingale, and hence a supermartingale under the probability measure $\overline{\mathrm{P}}$. Thus, using Eq. (B.1) in conjunction with the definition of the manager's indirect utility function we obtain that

$$
u(x, a) \leqslant U(x)\left\{\left(\alpha^{*}\right)^{-\Theta_{1}} h\left(\alpha^{*}\right) \sup _{\tau \in \mathscr{T}^{a}} \overline{\mathrm{E}}\left[L_{\tau}\right]\right\}^{1 / \Phi} \leqslant U(x)\left[h\left(\alpha^{*}\right)\left(\frac{a}{\alpha^{*}}\right)^{\Theta_{1}}\right]^{1 / \Phi} .
$$

Now let $\tau^{*}$ denote the first hitting time of the constant barrier $\alpha^{*}$ by the value of the underlying assets. Using the continuity of the latter process in conjunction with the fact that the initial value of the underlying assets is less that $I \leqslant \phi \alpha_{0}$, we obtain

$$
u(x, a) \geqslant U(x)\left\{\overline{\mathrm{E}}\left[\mathrm{e}^{-q \tau^{*}} h\left(A_{\tau^{*}}\right)\right]\right\}^{1 / \Phi}=U(x)\left[h\left(\alpha^{*}\right)\left(\frac{a}{\alpha^{*}}\right)^{\Theta_{1}}\right]^{1 / \Phi} .
$$


This contradicts Eq. (B.2) and hence establishes the optimality of the stopping time $\tau^{*}$ as well as the expression for the value function given in the statement of the theorem.

\section{Appendix C. A reduced-form model}

In this appendix we provide an explicit solution to the model using a reduced-form version of the specification presented in the main text. Notably, we presume that the proportional shock $\vartheta$ incurred by the manager at the time of investment is larger than one when the manager selects the value-maximizing policy and decreases with deviations from value-maximization in investment policy. In particular, we assume that

$$
\vartheta_{\tau}=\vartheta\left(A_{\tau}\right):=\Lambda\left[1_{\left\{\alpha \leqslant \alpha_{0}\right\}}\left(\frac{\phi \alpha-I}{\phi \alpha_{0}-I}\right)^{\gamma}+1_{\left\{\alpha>\alpha_{0}\right\}}\left(\frac{\phi \alpha_{0}-I}{\phi \alpha-I}\right)^{\gamma}\right],
$$

where $\Lambda \geqslant 1, \gamma \in(0,1)$ is a cost parameter, $\tau$ is the selected investment time, and $\alpha_{0}$ is the investment threshold that maximizes project value. Although simple, this specification captures two essential aspects of agency relationships. In the model, there is a benefit to following the value maximizing investment policy and the larger $\Lambda$ the larger this benefit. In addition, there is a cost to deviating from valuemaximization, and the larger $\gamma$ the larger this cost.

The following theorem provides a closed-form solution to the manager's utilitymaximization problem under this specification.

Theorem 3. Assume that $q$ is strictly positive, that $\Theta_{1}<\eta(1-R) \Phi$, and that the starting value of the assets is below the investment threshold. Then the manager's indirect utility function is given by

$$
u(x, a)=U(x) \overline{\mathrm{E}}\left[\mathrm{e}^{-q \tau^{*}} \vartheta\left(A_{\tau^{*}}\right)^{(1-R) \Phi}\right]^{1 / \Phi}=U(x) \vartheta\left(\alpha^{*}\right)^{(1-R)}\left(\frac{a}{\alpha^{*}}\right)^{\Theta_{1} / \Phi},
$$

where the optimal stopping time $\tau^{*}$ is the first hitting time of the constant investment threshold defined by

$$
\alpha^{*}:=\min \left\{\alpha_{0}, \frac{\Theta_{1}}{\Theta_{1}-\gamma(1-R) \Phi} \frac{I}{\phi}\right\},
$$

for $\Theta_{1}-\gamma(1-R) \Phi>0$ and $\alpha^{*}=\alpha_{0}$ otherwise.

This result shows explicitly that the trigger level selected by the manager is lower than the value-maximizing threshold and that the manager invests early compared with the value-maximizing investment policy. In addition, it demonstrates that the threshold selected by the manager decreases with risk aversion. The numerical results associated with this specification are quantitatively very close and qualitatively identical to those presented in the main text. 


\section{References}

Boyle, G., Guthrie, G., 2003. Investment, uncertainty, and liquidity. Journal of Finance 58, 2143-2166. Brennan, M., Schwartz, E., 1985. Evaluating natural resource investments. Journal of Business 58, $137-157$.

Descamps, J.-P., Mariotti, T., Villeneuve, S., 2004. Investment timing under incomplete information. Mathematics of Operations Research 30, 472-500.

Dixit, A., Pindyck, R., 1994. Investment Under Uncertainty. Princeton University Press, Princeton.

Duffie, D., 2003. Dynamic Asset Pricing Theory, third ed. Princeton University Press, Princeton.

Grenadier, S., 2002. Option exercise games: an application to the equilibrium investment strategies of firms. Review of Financial Studies 15, 691-721.

Grenadier, S., Wang, N., 2005. Incentives and investment timing: real options in a principal-agent setting. Journal of Financial Economics 75, 493-533.

Guo, X., Miao, J., Morellec, E., 2005. Irreversible investment with regime shifts. Journal of Economic Theory $122,37-59$.

Henderson, V., 2005. Valuing the option to invest in an incomplete market. Working Paper, Princeton University.

Hennessy, C., 2004. Tobin's Q, debt overhang, and investment. Journal of Finance 59, 1717-1742.

McDonald, R., Siegel, D., 1986. The value of waiting to invest. Quarterly Journal of Economics 101, 707-728.

Mello, A., Parsons, J., 2000. Hedging and liquidity. Review of Financial Studies 13, 127-153.

Miao, J., Wang, N., 2005. Investment, consumption, and hedging under incomplete markets. Working paper, Boston University.

Morellec, E., 2004. Can managerial discretion explain observed leverage ratios? Review of Financial Studies 17, 257-294.

Stulz, R., 1990. Managerial discretion and optimal financial policies. Journal of Financial Economics 26, $3-27$.

Zwiebel, J., 1996. Dynamic capital structure under managerial entrenchment. American Economic Review $86,1197-1215$. 